\title{
SOFTWARE PARA GERENCIAMENTO DE RISCO E SEGURANÇA DO PACIENTE COM IMPRESSÃO DE PULSEIRA DE IDENTIFICAÇÃO
}

\section{ARTIGO ORIGINAL}

REIS, Fabiana Candido dos ${ }^{1}$

REIS, Fabiana Candido dos. Software para Gerenciamento de Risco e Segurança do Paciente com Impressão de Pulseira de Identificação. Revista Científica Multidisciplinar Núcleo do Conhecimento. Ano 04, Ed. 08, Vol. 01, pp. 140-169. Agosto de 2019.

ISSN:

2448-0959,

Link

de

acesso: https://www.nucleodoconhecimento.com.br/saude/pulseira-de-identificacao

\section{RESUMO}

A segurança do paciente é foco de discussões no mundo todo, durante a última década, e é parte dos critérios básicos para garantir-se a qualidade de assistência ao paciente, logo a adoção de estratégias para redução de falhas e eventos danosos em instituições de saúde é fundamental. Considerando o gerenciamento de risco informatizado um instrumento de apoio para as tomadas de decisões do enfermeiro, para registro dos riscos identificados e auxílio na identificação de outros, além de apresentar um banco de dados rico em informações para pesquisa. Este estudo teve como objetivo desenvolver um software para gerenciamento de riscos e impressão de pulseira única de identificação com sinalização de alerta de riscos para segurança assistencial e prevenção de eventos adversos durante a permanência do paciente no serviço de saúde. Trata-se de uma pesquisa aplicada e de produção tecnológica $O$ desenvolvimento deste estudo obedece a algumas fases de um ciclo de vida de um software. Para a construção deste software foi utilizado na fase de planejamento a metodologia Scrum, na fase de programação foi utilizado a linguagem de HTML (HyperText Markup Language), XML (eXtensible Markup Language) e PHP (Personal

1 Mestranda do Programa de Mestrado Profissional do processo de cuidar, Especialista em Segurança do Paciente, Graduação em Enfermagem. 
Home Page Tools) foram escolhidas porque sua sintaxe é bastante simples. O softaware de gerenciamento de risco informatizado para a segurança do paciente tem por foco inserir dados de registros a respeito do paciente e imprimir a pulseira única de identificação com a cor da tarja correspondente para sinalização dos alertas e atenção de riscos. O sistema é composto de 36 telas de acesso e está estruturado em quatro módulos, são eles: o módulo de classificação de risco pronto atendimento é composto pelo protocolo de Acolhimento ACCR; o módulo alerta de riscos contém 17 alertas de risco e atenção e as escalas de avaliação; o módulo informações é composto por uma lista de fatores de risco em cada alerta tipo checklist para seleção e registros com espaços em branco para descrição de dados e individualização da assistência; o módulo visualizar pulseira apresenta a ilustração da pulseira com os dados registrados previamente pelo enfermeiro. Buscou-se com o desenvolvimento do software a elaboração da pulseira única de identificação com a classificação e/ou alertas de riscos e cuidados especiais e informações visíveis em forma de código de cores tarjadas, através do gerenciamento de risco.

Palavras-Chave: enfermagem, gestão de riscos, informática em enfermagem, segurança do paciente, sistemas de identificação de pacientes.

\section{INTRODUÇÃO}

A segurança do paciente é pauta de discussões em todo o mundo desde a última década, e é um dos processos básicos para asseverar a qualidade da assistência ao paciente, portanto a adoção de estratégias para redução de erros e eventos adversos em instituições de saúde é fundamental (SANTOS, 2014). O resguardo do paciente nas instituições de saúde são responsabilidades de cunho emergentes e tem sido foco de discussões em âmbito mundial (PEREIRA; SOUZA; FERRAZ, 2014).

Brasil (2014) pontua que o impulso em prol da segurança do paciente nasceu no fim do século XX, logo após a publicação do relatório do Institute of Medicine (IOM) dos Estados Unidos da América (EUA), titulado como "Errar é humano: construindo um sistema de saúde mais seguro" (To err is Human: building a safer health system), em 1999, que apresentou os resultados de vários estudos constatando que entre 44.000 
e 98.000 pacientes morriam a cada ano nos hospitais do referido país em virtude dos danos causados pelo cuidado à saúde.

Esta publicação pode ser considerada como marco da confluência acerca desta temática, pois a partir dela surgiu a era da segurança mobilizando muitos eventos e estudos sobre segurança do paciente (BRASIL, 2014).

Ao traçar a trajetória sobre a história e as transformações da segurança do paciente, Nascimento e Draganov (2015) constataram que desde tempos remotos, ainda que incipiente, já havia uma responsabilidade acerca da temática. Constataram que o marco inicial deu - se com Florence Nightingale em 1852, enfermeira inglesa que foi trabalhar na Guerra da Criméia (1853 a 1856) e observando as condições precárias em que os soldados se encontravam, priorizou a segurança dos soldados como fator fundamental para uma boa qualidade nos cuidados prestados.

Em outubro de 2004, a Organização Mundial da Saúde (OMS) lançou formalmente na 57 Assembleia Mundial da Saúde, a Aliança Mundial para Segurança do Paciente. Com o intuito de minimizar as consequências adversas da assistência à saúde insegura e favorecer normas e práticas de políticas de segurança do paciente, formulou os Desafios Globais para a Segurança do Paciente, para uma ação de cunho internacional (WHO, 2005).

A recomendação da identidade correta do paciente foi empregada pela OMS como a meta internacional inicial de segurança do paciente em nível mundial e passou a ser considerada fundamento do cuidado seguro. Consiste na utilização de identificações, como pulseiras, em pacientes hospitalizados, em observação nas unidades de pronto atendimento, adultos e crianças, sob qualquer condição de assistência. São procedimentos essenciais à prevenção de danos durante o cuidado à saúde (REBRAENSP, 2013).

Toda equipe multiprofissional, incluindo a de enfermagem, é responsável pela confirmação e verificação da identificação do paciente durante o período que o mesmo permanecer sob os cuidados da instituição (BRASIL, 2013). 
Para desenvolver seu trabalho, a enfermagem utiliza como base a informação, portanto faz-se necessário compreender o que a tecnologia da informação pode modificar o seu trabalho diário e para criar novas oportunidades, além de direcionar a enfermagem de forma a ocupar seu espaço frente aos processos de mudanças (SPERANDIO, 2002).

Santos (2010) constatou que a informática se tornou relevante para o processo de trabalho, tanto assistencial, quanto gerencial para a enfermagem, pois permite registro e acesso rápidos às informações dos pacientes, bem como minimiza erros e facilita a comunicação entre os profissionais.

Edwards (2009) enfatizou que, para que o acesso à informação seja possível, ela é armazenada e organizada no software, em um arquivo denominado banco de dados. Pressman (2011) confirmou que o banco de dados contém uma coleção de informações devidamente organizada à qual se tem acesso por intermédio do software.

Partindo deste princípio, a seguinte questão de pesquisa foi proposta: "É possível construir um software para gerenciamento de riscos com impressão de uma pulseira única de identificação do paciente capaz de sinalizar os alertas de riscos?

Portanto, nossa proposta é de um programa computacional específico para gerenciamento de risco a partir de um protótipo já desenvolvido que possa beneficiar a equipe multiprofissional a favor da segurança do paciente e, um único meio de dispositivo de identificação, sendo este a pulseira, possa de maneira prática, segura e confortável contribuir para a identificação do paciente, bem como com a sinalização dos riscos e situações peculiares que inspiram uma atenção especial ao qual o mesmo está exposto no atendimento em serviços de saúde. 


\section{OBJETIVO}

Desenvolver um software para gerenciamento da segurança do paciente com a impressão de uma pulseira única de identificação e sinalização de alerta de riscos a partir de um software protótipo.

\section{MATERIAL E MÉTODO}

\section{TIPO DE ESTUDO}

Trata-se de uma pesquisa aplicada e de produção tecnológica para elaboração de um software para gerenciamento de riscos assistenciais com proposta de impressão de pulseira única para identificação do paciente e sinalização de riscos para segurança assistencial de enfermagem em relação à precaução de ocorrências adversas durante a permanência do paciente no serviço de saúde.

Como pesquisa aplicada e de produção tecnológica entende-se a geração de novos conhecimentos e processos, para a solução de problemas específicos com objetivo prático e operacionalização das ideias. A pesquisa tecnológica parte de um conhecimento pré-existente e, através da pesquisa e/ou experiência prática visa à materialização de um produto, protótipo, processo ou instalação piloto (GIL, 2010; MAGALHÃES, 2005; SOUZA, 2013).

\section{PROCEDIMENTOS METODOLÓGICOS}

O desenvolvimento deste estudo obedece a algumas fases de um ciclo de vida de um software, sendo a primeira fase a construção de um protótipo e subsequente o desenvolvimento através da programação.

Software é definido por Pressman (2011) como sendo um conjunto de documentos que tem como objetivo mostrar como se dá a operação e o uso dos programas.

Sommerville (2008) refere que há dois modelos fundamentais de software, o software genérico que é sistema desenvolvido e colocado à venda para aquisição de qualquer 
usuário, como por exemplo processadores de texto e o software personalizado que é desenvolvido para um objetivo e cliente específicos.

Todo o software tem um ciclo de vida, que pode ser considerado como uma sequência de fases e tarefas, que serão executadas no decorrer do desenvolvimento de um sistema, isto é, o software passa por inúmeras fases desde o início, com a geração de ideias até a sua implantação (PRESSMAN, 2011).

Para exemplificar estas fases, tomamos um modelo denominado cascata, que se desenvolve em cinco fases, são elas: definição de requisitos, projeto de sistemas e software, implantação e teste de unidade, integração e teste de sistema e por fim operação e manutenção, lembrando que a fase seguinte só acontece se a anterior foi finalizada (SOMMERVILLE, 2008).

\section{PROTOTIPAÇÃO}

Para o desenvolvimento de um software é necessário escolher uma metodologia ou modelo. Existe uma gama deles, mas sua escolha se dá a partir da definição da interatividade com o usuário e da quantidade de documentação que deverá ser desenvolvida. Dentre as metodologias mais utilizadas tem-se: modelo cascata, RUP (Rational Unified Process), modelo espiral, evolucionário, modelo iterativo incremental, metodologia extreme programming (XP) e modelo prototipagem ou prototipação (PRESMAN, 2011; SOMMMERVILLE, 2008).

Sommerville (2008) define a prototipação de um sistema como sendo uma versão rápida ou parte de um sistema, que é desenvolvido rapidamente, com o objetivo de verificar as necessidades do cliente, bem como a viabilidade de algumas decisões do projeto. Esta versão do sistema permite aos usuários experimentarem antes da entrega e com isso é possível refinar seus requisitos. Então, um protótipo é considerado uma versão protótipo de um sistema de software, que será utilizado para apresentar conceitos, experimentar opções e identificar problemas e soluções. 
As vantagens do modelo de prototipação é a possibilidade de obter uma versão do que será o sistema e demonstrar sua viabilidade, com um pequeno investimento inicial, a experiência adquirida no desenvolvimento do protótipo será de extrema utilidade permitindo a redução de custos nas fases posteriores e resultando num sistema melhor concebido, enquanto que os problemas da prototipação estão relacionados à reconstrução, o cliente exige alguns acertos no protótipo para torná-lo um produto, as recorrentes mudanças tendem a prejudicar sua estrutura. Agregar mudanças futuras do software torna-se cada vez mais dificultoso (PRESSMAN, 2011).

Pressman (2011) propõe o desenvolvimento de um protótipo em seis fases, são elas: coleta e refinamento dos requisitos, projeto rápido, construção do protótipo, avaliação do protótipo, refinamento do protótipo e engenharia de projeto.

Neste estudo foram utilizadas as três primeiras fases de desenvolvimento do protótipo. $\mathrm{Na}$ primeira fase ou fase de coleta e refinamento de requisitos foram discutidas as ideias, elencados os objetivos e o conteúdo do software.

Os riscos seriam sinalizados por meio de tarjas coloridas de forma discreta, sem expor o paciente, não sendo necessário o uso de mais de uma ou até várias pulseiras coloridas para identificação dos alertas de riscos, como pode se observar, atualmente, nos serviços de saúde, e que muitas vezes dificulta recolocação das mesmas em casos que necessitam a retirada para realizar procedimentos como punções e inserções de cateteres; alguns pacientes pelo desconforto se recusam a usar as pulseiras avulsas, podendo ocasionar aumento no risco de incidentes durante a assistência à saúde.

Outro fator que fala a favor da construção de um dispositivo único é que diariamente o enfermeiro avalia as ameaças aos quais os pacientes estão expostos e pode acrescentar ou retirar um risco identificado e com esta proposta não seria necessário introduzir mais uma pulseira e sim uma tarja com a cor correspondente ao risco identificado e no caso da remoção do alerta de risco, identificado anteriormente, seria então retirada a tarja colorida correspondente ao mesmo. 
A estrutura proposta para este dispositivo se compõe de uma pulseira branca que possui espaço para inserção das informações do paciente, como nome completo, em crianças acrescentar o nome completo da mãe, data de nascimento, registro hospitalar, código de barras e/ou QR ${ }^{\circledR}$ code e as tarjas na cor correspondente ao alerta de risco. As cores poderiam ser inseridas de acordo com a necessidade apresentada ou relatada pelo paciente, ou seja, quantas forem necessárias, esta solução evitaria o desconforto por meio do uso de várias pulseiras, reduziria gastos, além de proporcionar uma visualização organizada facilitando e otimizando o procedimento de trabalho da equipe de saúde, colaborando também com a redução dos resíduos sólidos de serviços de saúde.

A partir da ideia do modelo de pulseira única de identificação e sinalização de riscos surgiu uma segunda ideia necessária para concretização do modelo que foi o desenvolvimento de um software para gerenciamento de risco de pacientes, tanto internado como em espera para atendimento em setores como ambulatório e pronto socorro com a confecção e impressão da pulseira única, a partir do gerenciamento de risco.

Com relação à classificação de risco em unidades de pronto socorro foram destacados dois instrumentos, o acolhimento com classificação de risco ACCR e a classificação de risco de Manchester.

Para o gerenciamento de riscos foram selecionados 17 alertas, sendo 14 de riscos e três alertas de atenção. Os alertas de riscos são: risco de queda, risco de alergia, risco de lesão por pressão, risco de reação transfusional, risco de broncoaspiração, risco de fuga, risco de transmissibilidade, risco de extubação acidental, risco para flebite, risco para infecção, risco para sangramento, risco para tromboembolismo pulmonar e trombose venosa profunda, risco para exteriorização acidental de sonda nasoenteral e risco para suicídio. Já os alertas de atenção são: ser praticante da denominação religiosa -Testemunha de Jeová, possuir necessidade de restrição de manipulação de membros e estar em cuidados paliativos, foram considerados estes alertas pois são de extrema importância na tomada de decisão assistencial. 
Foram utilizados os indicadores de qualidade propostos pelo Núcleo de Apoio à Gestão Hospitalar (NAGEH) um subgrupo do Compromisso com a Qualidade Hospitalar (CQH) mantido pela Associação Paulista de Medicina (APM) e pelo Conselho Regional de Medicina do Estado de São Paulo (CREMESP), são empregados como guia para acompanhar e avaliar a assistência e as atividades de um serviço e são, ainda, compreendidos como dados de desempenho de processos específicos, produtos e da organização como um todo (NAGEH, 2012).

Foi utilizada a Taxonomia da NANDA, pois os diagnósticos de enfermagem também subsidiaram o conteúdo relativo aos riscos, considerando que o diagnóstico de enfermagem é o parecer clínico sobre a conclusão do indivíduo, família ou comunidade aos problemas de saúde reais e/ou potenciais, sendo fornecedor da base para a escolha de ações para alcançar os efeitos aguardados pelos quais o enfermeiro é responsável (NANDA, 2015; COFEN, 2009). É a expressão de uma situação do indivíduo que, em nosso julgamento, necessita de intervenção de enfermagem.

$\mathrm{Na}$ segunda fase, foi elaborado o projeto rápido. A partir da seleção do referencial teórico e do estabelecimento dos requisitos de como deveria funcionar o sistema, foi construído o layout das telas no Microsoft Office Power Point para o esboço do sistema.

A partir deste esboço partiu-se para a discussão com um profissional de informática com formação em análise de sistemas para a apresentação da proposta e construção do software- protótipo.

A terceira fase também denominada de construção do protótipo se iniciou com o estabelecimento de uma visão geral do software, com identificação de funções primárias que deveria realizar.

Para a construção deste protótipo foram utilizadas as linguagens de programação HTML (Hyper Text Markup Language), XML (eXtensible Markup Language), Java Script e CSS (Cascading Style Sheet) para o estilo das páginas. 
O HTML ${ }^{\circledR}$ é uma linguagem usada para expor e definir o conteúdo de uma página da Web em um formato estruturado, a única que o navegador consegue interpretar para a exibição de conteúdo e o XML é extremamente flexível e facilmente manipulado pelas aplicações de software, permite adaptações da estrutura de um documento a qualquer situação específica, essas linguagens foram escolhidas nesse estudo porque sua sintaxe é bastante simples (QUIERELLE, 2012; SILVA, 2010).

As chamadas folhas de estilo cascata, denominada CSS são usadas para descrever e personalizar a aparência do layout do conteúdo da Web, serve para editar o contento das páginas, tal a como cor de fundo, os estilos de texto, o distribuição do conteúdo e imagens. Assim, os CSS são arquétipos de formatação do documento HTML, utilizados mediante aos códigos de HTML (QUIERELLE, 2012).

O sistema foi desenvolvido para funcionar em plataforma Microsoft Windows. O Java Script é a linguagem de programação que é executada no navegador, é usado para criar sites e aplicativos interativos para usuários avançados. (SILVA, 2010).

O Java Script é a camada de desenvolvimento responsável por toda a movimentação das imagens o aparecimento do menu e do sub menu com vários outros itens do programa, é utilizada para controlar as linguagens de informação HTML e de formatação CSS. É um excelente recurso para executar rotinas do usuário. Por esse motivo utilizou-se o Java Script nesse projeto (SILVA, 2010).

Para registro das informações durante o gerenciamento de risco foram elaboradas questões do tipo "checklist", pois facilita a sistematização, o processo de avaliação e identificação dos alertas de risco. Para individualizar a assistência foram também incluídos campos de descrição / escrita livre. 


\section{PLANEJAMENTO, DESENVOLVIMENTO E FUNCIONALIDADE DO SOFTWARE}

A partir do modelo proposto na fase de prototipação partiu-se para a discussão com um profissional de informática com formação em análise de sistemas para a apresentação da proposta, programação e desenvolvimento software.

Para a construção do software a partir da prototipação seguiram-se as etapas de planejamento, desenvolvimento e funcionalidade.

\section{PLANEJAMENTO}

$\mathrm{Na}$ etapa de planejamento foi realizado uma análise conjunta onde foi elaborado um escopo para organização do desenvolvimento do projeto. Estruturado um modelo de reuniões semanais em quatro datas, sendo planejado como pauta de cada encontro: apresentação e explicação do protótipo por parte do mestrando, apresentação de proposta orçamentaria e tempo para desenvolvimento do software, aprovação do orçamento pelo mestrando e discussão da metodologia ágil para desenvolvimento do software por parte do programador, por último deu-se a assinatura do contrato e entrega de cronograma para início das atividades na semana subsequente.

Cabe salientar que a metodologia proposta para construção do software será a Scrum, gestão ágil para projetos deste segmento. Scrum é um framework rápido, fácil e leve, usado para a gestão da expansão de produtos complexos integrados a ambientes complexos. Scrum faz uso de uma interpelação iterativa e incremental para agregar valor regularidade, reduzindo os riscos do projeto (SABBAGH, 2017).

Rubin (2018) demonstra que no Scrum, os ciclos são chamados de Sprints. Eles portam dimensão fixa e sucedem um após do outro, sem interrupções entre eles, ou seja, tudo em um projeto que utiliza Scrum acontece dentro dos Sprints. Cada Sprint é composto por:

- Reunião de Sprint Planning, onde se realiza o planejamento do Sprint; 
- Trabalho de desenvolvimento do produto, que inclui tudo que é necessário para se entregar uma parte do produto pronta ao nal do Sprint;

- Reunião de Daily Scrum, integrada no cotidiano de desenvolvimento, que é uma reunião diária para gerar visibilidade e planejar o próximo dia de desenvolvimento;

- Reunião de Sprint Review, no qual os resultados do desenvolvimento realizado no Sprint são demonstrados aos clientes e demais interessados, realizando o oferecimento de seu feedback;

- Reunião de Sprint Retrospective, onde a equipe julga o que foi bem e o que necessita de melhorias na sua forma de trabalho;

- Quaisquer reuniões ou sessões programadas adicionais que se fizerem necessárias, a vista de exemplo, a reunião de Release Planning, e as sessões de Refinamento do Product Backlog.

A figura $E$ representa a interação do Scrum, indicando a sucessão de elementos presentes em todos os ciclos (ou Sprints) e a sucessão de ciclos.

Figura A: Interação ou sucessão de ciclos (Sprints) do Scrum

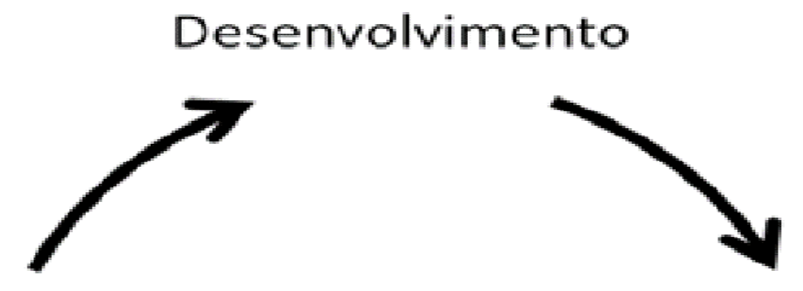

Sprint Planning
Sprint Review

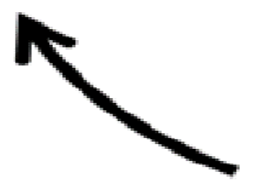

\section{Sprint}

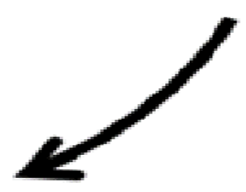

Retrospective

Fonte: Autor. 
O Sprint inicializa com a reunião de Sprint Planning, na qual se planifica o trabalho a ser efetuado no próprio Sprint. Nessa reunião, o Time de Desenvolvimento e Product Owner acordam a partir dos itens do alto do Product Backlog, sobre o que será definitivamente desenvolvido. Ou seja, simplificados pelo ScrumMaster, seleciona-se um conjunto de tópicos do alto do Product Backlog que se propõem capacidade de desenvolvimento durante o Sprint, uma antevisão, e estabelecem um uma meta de negócios a ser atingida com a prosperidade desses itens, chamada de Meta do Sprint. Assim, o Time de Desenvolvimento se compromete em atingir a Meta do Sprint (KNAPP et.al, 2017).

O Time de Desenvolvimento se encontra por no máximo quinze minutos em cada dia de trabalho de desenvolvimento, no mesmo horário e local, para a reunião de Daily Scrum. O objetivo do encontro é garantir a transparência e visibilidade de seu trabalho entre si e planejar, ainda que informalmente, o próximo dia de produção (RUBIN, 2018).

O feedback arquivado dos clientes, o mestrando na reunião de Sprint Review no caso, é requerido pelo Product Owner como matéria-prima para alterações no Product Backlog - para mudar o produto em processo de geração, buscando melhor atender às necessidades dos consumidores (SABBAGH, 2017)

O Sprint Backlog é uma lista de itens selecionados do alto do Product Backlog para o desenvolvimento do Incremento do Produto no Sprint(oquê),adicionada de um plano de como esse trabalho será realizado(como). O refinamento do Product Backlog é o ato de adicionar detalhes, estimativas e pedidos a itens no Product Backlog (KNAPP et.al, 2017). 
Figura B: O ciclo do Scrum

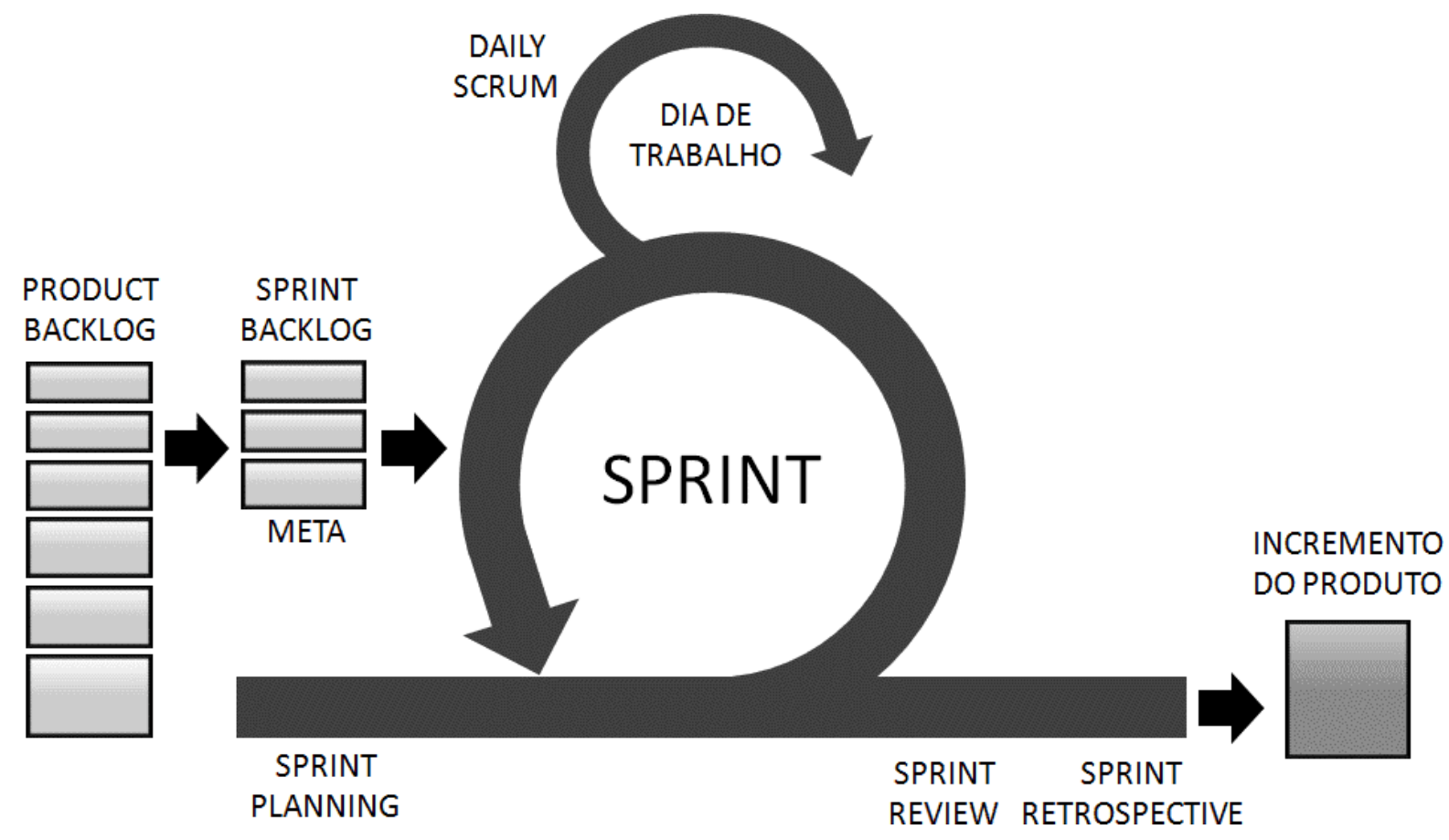

Fonte: Autor.

Por via de Releases regulares, o Time de Scrum obtém feedback dos usuários do produto e podem assim vir a reduzir os riscos e produzir o produto adequado. O Time de Scrum também possibilita um senso de progresso do projeto aos seus clientes e interessados, podendo dispor gratificação ao investimento realizado pelos clientes do projeto (RUBIN, 2018).

Rubin (2018) afirma que pode haver uma reunião de Release Planning para cada Release, sendo esta realizada em dado momento antes do início dos trabalhos para a Release correspondente, o que deve acontecer durante o último Sprint do Release.

\section{DESENVOLVIMENTO}

PHP $^{\circledR}$ ou PHP Hypertext Preprocessor, quer dizer que o produto foi inicialmente titulado de "Personal Home Page Tools"; e ao crescercom uma meta, um nome novo e mais adequado foi eleito por votação da comunidade. A dimensão ".php" é a aconselhável para designar um arquivo $\mathrm{PHP}^{\circledR}$ (SKLAR, 2004). 
$\mathrm{PHP}^{\circledR}$ é uma linguagem de criação de scripts incrustrado em $\mathrm{HTML}^{\circledR}$ no servidor. O PHP pode ser considerado como uma coleção de supertags de $\mathrm{HTML}^{\circledR}$ que adicionam encargos do servidor às suas páginas da Web. É possível fazer uso do PHP para construir imediatamente uma complexa página da Web (SOARES, 2013).

O PHP $^{\circledR}$ não obtém relação direta com layout, eventos ou tudo o que envolva a aparência de uma página da Web. Grande parte do que é realizado pelo PHP é invisível para o usuário final. Quando se visualiza uma página de PHP, não será capaz de dizer que não foi escrita em $\mathrm{HTML}^{\circledR}$, pois o resultado final do $\mathrm{PHP}^{\circledR}$ é $H T M L^{\circledR}$.

O HTML ${ }^{\circledR}$ é uma linguagem usada para expor e definir o conteúdo de uma página da Web em um formato estruturado, a única que o navegador consegue interpretar para a exibição de conteúdo e o XML é extremamente flexível e facilmente manipulado pelas aplicações de software, permite adaptações da estrutura de um documento a qualquer situação específica, essas linguagens foram escolhidas nesse estudo porque sua sintaxe é bastante simples (QUIERELLE, 2012; SILVA, 2010).

\section{SOFTWARE}

Software é o conjunto modificável de instruções, ordenadas e lógicas, direcionadas ao hardware para a execução de processos requeridos à solução de procedimentos necessários, à solução dos problemas e tarefas do processamento de dados. As classificações de software estão divididas quanto a finalidade de uso, sendo básico, aplicativo/aplicação e linguagem de programação (SOMMERVILLE, 2011).

\section{PRODUTO FINAL}

O produto final se compõe de uma pulseira branca que possui espaço para inserção das informações do paciente, como nome completo, em crianças acrescentar o nome completo da mãe, data de nascimento, registro hospitalar, código de barras e/ou $\mathrm{QR}^{\circledR}$ code e as tarjas na cor correspondente ao alerta de risco. As cores poderiam ser inseridas de acordo com a necessidade apresentada ou relatada pelo paciente, ou seja, quantas forem necessárias. 


\section{RESULTADOS}

\section{GERIS - SOFTWARE PARA GERENCIAMENTO DE RISCOS} ASSISTENCIAIS COM PROPOSTA DE IMPRESSÃO DE PULSEIRA ÚNICA

O nome proposto para o software foi GERIS, que advém das iniciais das palavras Gerenciamento de Risco. O sistema consiste num programa de computador com o objetivo de apoiar o enfermeiro na tomada de decisão durante a assistência ao indivíduo através do mapeamento de riscos assistenciais.

Para elaboração do projeto houve inicialmente uma fase de coleta de dados e definições de requisitos desenvolvidos pela autora do protótipo. O conteúdo do protótipo está fundamentado em instrumentos e ferramentas relacionados à prática assistencial de Enfermagem, como indicadores de qualidade; diagnósticos de enfermagem e documentos oficiais emitidos pelo Ministério da Saúde, bem como outras literaturas encontradas em bases de dados e livros especializados.

Quadro 01 - Fundamentação teórica para a construção dos instrumentos e alerta de riscos

\begin{tabular}{|l|l|l|}
\hline Informações & BRASIL (2013, 2014a, 2013b); \\
& FIOCRUZ/Proqualis(2014); Gomes (2008); \\
& Hinrichsen (2011); Manual de Indicadores de \\
& Enfermagem NAGEH (2012); NANDA (2015); Lima, \\
& Melleiro (2011), Oliveira(2014). Siqueira (2015); \\
Zárate-Graiales. et al.(2015); ANVISA (2013); & American hospitalassociation (2008); ABNT (2010); \\
Barcelos (2016); Costa, Meirelles, Erdmann(2013); & Fassini (2012);Fawkes (2007) Hemesath \\
& (2015)Hoffmeister, Moura (2015); Milagres (2015); \\
& Neves,Melgaço (2001); Norris, Ranger (2009); OMS
\end{tabular}


(2010); Pimenta et al. (2015); Public health and clinical systems (2014); Santos (2014); Smith et al. (2011); Sperandio (2002, 2008); Silva (2013); WHO(2007b);


Risco de extubação NAGEH (2012); Barcellos et al. (2016); Carvalho (2010); acidental Castellões, Silva, (2007, 2009); Duarte(2016); Neto et al. (2014)

Risco de fuga Vieira; Dall'agnol (2009); Vieira (2005); COREN(2010b); Risco de lesão por NAGEH (2012), NANDA (2015); Laurenti et al.(2015); pressão Lyder, Ayello (2008); Paranhos (1999); Freitas, Alberti (2013); SANTOS et al. (2015);

Risco de queda NAGEH (2012); Severo et al., (2014); NANDA(2015); Urbanato (2013); American geriatrics society (2010); Sociedade brasileira de geriatria egerontologia (2008);

Risco de reação BRASIL (2010)

transfusional

Risco transmissibilidade

Risco

exteriorização

acidentalde

sondanasoeteral

Risco para flebite

Risco para infecção de Lacerda (2014); BRASIL (2010); Andrade (2013);
NAGEH (2012);Milutinović, Simin, Zec (2015); Marinho (2011); Tertuliano (2014); Ray-Barruel etal. (2014); Almeida (2014); NANDA (2015); 


\begin{tabular}{|c|c|}
\hline $\begin{array}{ll}\text { Risco } & \text { para } \\
\text { sangramento }\end{array}$ & NANDA (2010) \\
\hline $\begin{array}{l}\text { Risco para } \\
\text { tromboembolismo } \\
\text { pulmonare trombose } \\
\text { venosa profunda }\end{array}$ & $\begin{array}{l}\text { Caramelli et al., (2004); Okuhara et al. (2015);SBACV } \\
\text { (2015); Veiga et al. (2015) }\end{array}$ \\
\hline Testemunha de Jeová & França (2008); Takaschima (2016); COREN (2013); \\
\hline Restrição de membros & BRASIL (2016); \\
\hline Cuidados paliativos & $\begin{array}{l}\text { CREMESP, 2008; Faller (2016); Guimarães (2016); } \\
\text { Lima, Oliveira (2015); Oliveira et al. (2016) Gomes, } \\
\text { Othero (2016);Evangelista et al. (2016); BRASIL (2002); } \\
\text { Santos, Oliveira, Feijao (2016); WHO(2002); }\end{array}$ \\
\hline Risco para Suicídio & 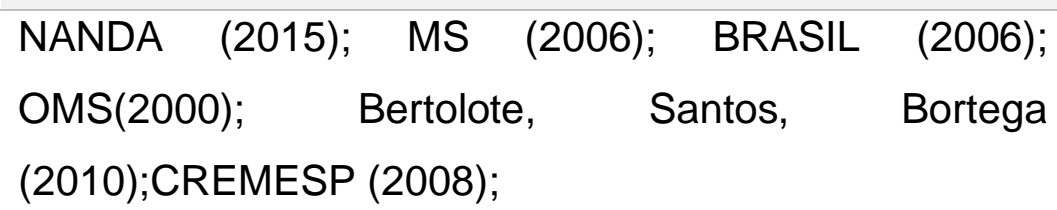 \\
\hline
\end{tabular}

Fonte: Santana, 2017 - Desenvolvimento de Software Protótipo para Gerenciamento de Risco e Segurança do Paciente com Impressão de Pulseira de Identificação.

Para compor o sistema de gerenciamento de riscos e segurança do paciente, denominado GERIS foram selecionados 17 alertas, sendo 14 de riscos e três alertas de atenção.

Os alertas de riscos foram: risco de queda, risco de alergia, risco de lesão por pressão, risco de reação transfusional, risco de broncoaspiração, risco de fuga, risco de transmissibilidade, risco de extubação acidental, risco para flebite, risco para infecção, risco para sangramento, risco para tromboembolismo pulmonar e trombose venosa profunda, risco para exteriorização acidental de sonda nasoenteral e risco para suicídio. Já os alertas de atenção são: ser praticante da denominação religiosa Testemunha de Jeová, possuir necessidade de restrição de manipulação de membros e estar em cuidados paliativos, foram considerados estes alertas pois são de extrema importância na tomada de decisão assistencial. 
$\mathrm{Na}$ composição dos alertas ainda foram incluídas escalas de riscos assistenciais para fundamentar a identificação dos riscos ou de atenção.

O sistema contém 36 telas, que se apresentarão da seguinte maneira:

1. Telas de acesso e cadastro do profissional -04 telas

2. Tela do menu inicial -01 tela

3. Telas de busca e cadastro do paciente -03 telas

4. Telas Módulo Classificação de Risco - Pronto Atendimento e Riscos Assistenciais - 02 telas

5. Telas Módulo Alerta de Riscos - 01 tela

6. Telas Módulo Informações de alertas e atenção de riscos e as escalas de avaliação - 24 telas

7. Telas Visualizar Pulseira - 01 tela

As informações dos ícones do "menu de paciente" foram pré-estabelecidas previamente, para que o usuário possa acessá-las apenas com um toque no dispositivo de entrada (mouse).

Para o desenvolvimento do software foi obedecido a lógica apresentada no diagrama abaixo: 
Figura C: Diagrama do software GERIS

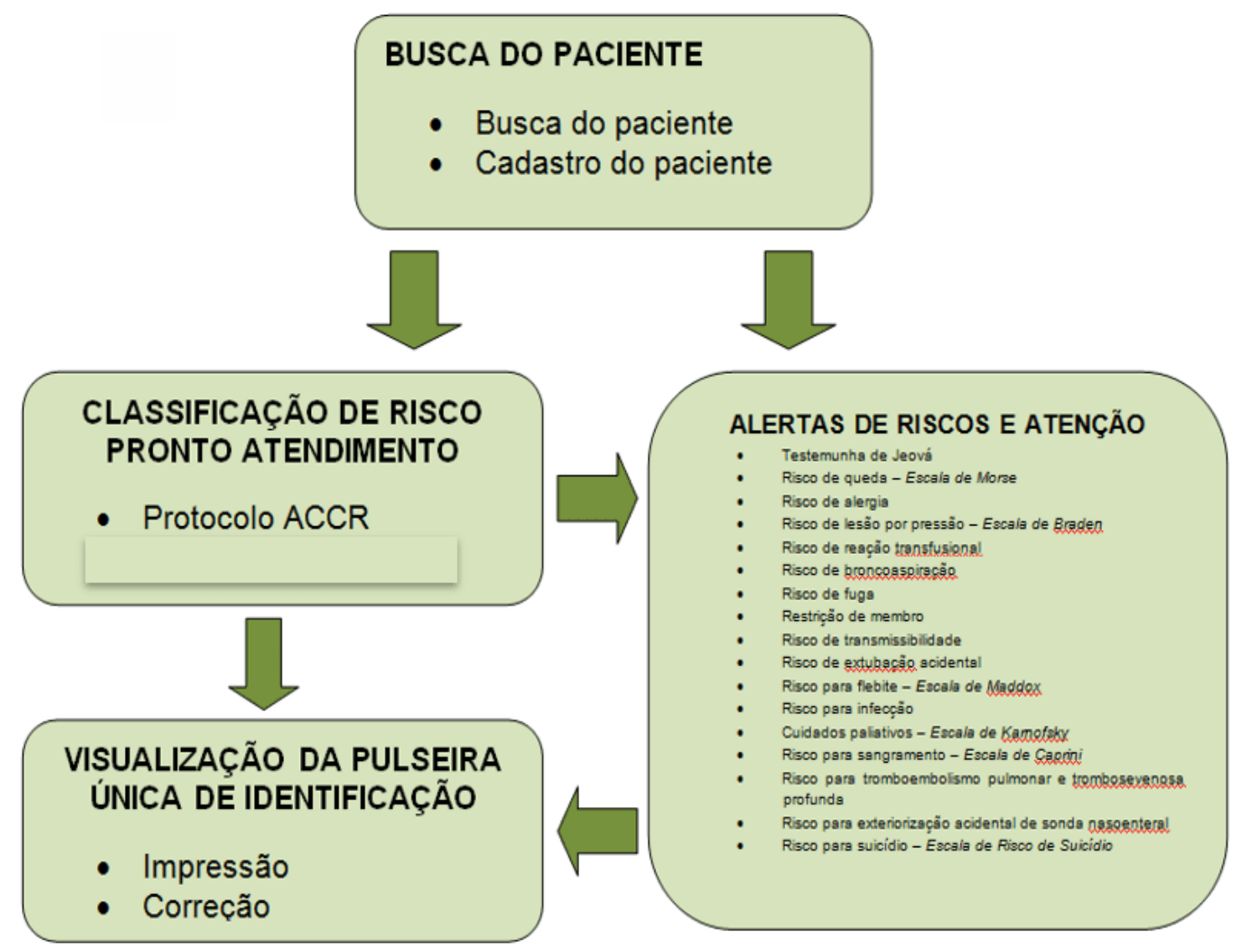

Fonte: Autor.

No Módulo Classificação de Risco - Pronto Atendimento o sistema será estruturado em fases referentes ao processo de classificação e gerenciamento de risco. As fases denominadas: Classificação de Risco para Atendimento em Pronto Socorro contém o protocolo de Manchester e o de Acolhimento com classificação de risco, proposto pelo Ministério da Saúde, com opções de atendimento segundo os sinais e sintomas do indivíduo.

No Módulo Alerta de Riscos serão desenhados a identificação e seleção dos alertas com probabilidade de causar incidentes durante a assistência no serviço de saúde, e o ícone de cadastro de informações específicas referentes aos riscos, com opção de avaliação de escalas de riscos, viabilizando a elaboração dos alertas de riscos que deverão ser gerenciados pelo enfermeiro, por meio da utilização dessa base de dados 
previamente estabelecida pelo sistema, e gerar, automaticamente a pulseira de identificação com as tarjas nas cores correspondentes.

No Módulo Informações será listado os alertas de risco e atenção para o enfermeiro selecionar os alertas adequados e inserir os dados referentes ao mesmo, conforme o checklist pré-estabelecido, será optado por itens de seleção para proporcionar ao enfermeiro menos tempo com o papel e maior disponibilidade a assistência direta ao paciente, esses registros poderão ficar armazenados e serem consultados e atualizados, oferece também dados para fins de pesquisa estatística. Alguns alertas terão o complemento da escala de avaliação de risco para registro do escore.

No Módulo Visualizar Pulseira após o enfermeiro ter feito a classificação de risco ou a seleção dos alertas de risco ou até mesmo ambas para o mesmo paciente ele poderá ver a ilustração da pulseira única com as cores das tarjas escolhidas para conferência dos dados registrados e impressão após confirmação ou optar pela correção no ícone apropriado.

As telas serão desenvolvidas com cores leves e padronizadas, as tarefas com informações diferentes serão divididas em diferentes telas, para facilitar a memorização do usuário, e essa separação será feita com o uso de ícones de entrada. O menu apresentado contém opções referentes as informações dos conteúdos.

Figura D: Tela de acesso do profissional

A tela de acesso profissional tem o objetivo de garantir acessibilidade segura ao sistema, considerando os requisitos de rastreabilidade relacionado a data, horário e acesso nominal do usuário. Conforme citado na Figura J, o cadastro prévio permitirá a confecção de login e senha individual permitindo o acesso ao software. 


\section{2दि? GERIS}

\section{GERIS - Gerenciamento de risco}

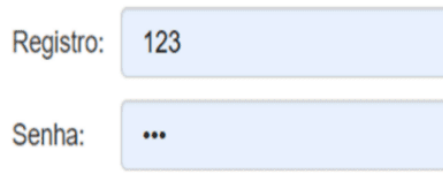

Esquecia senha

Fonte: Autor.

Figura E: Tela Menu Paciente: módulos

Cadastro de pacientes

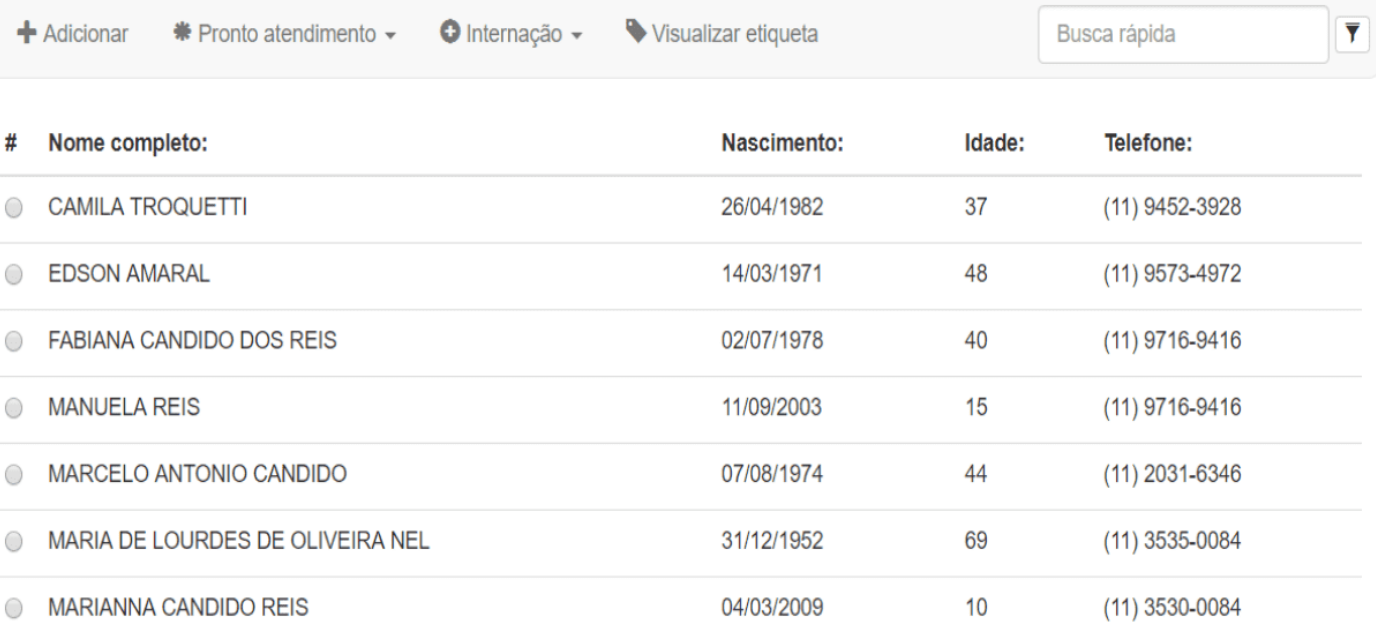

Fonte: Autor.

RC: 35278

Disponível em: https://www.nucleodoconhecimento.com.br/saude/pulseira-de-identificacao 
Figura F: Tela Pronto Atendimento: Classificação de Risco ACCR

Ao selecionar o ícone "pronto atendimento" na tela menu paciente: módulos (ver figura N) é possível clicar no ícone ACCR para realizar a classificação de risco para pacientes admitidos no pronto atendimento. Na tela pronto atendimento: classificação de risco ACCR faz-se necessário completar as seguintes informações na tela em questão: número do prontuário, convênio público ou privado e data e hora da admissão.

Ao término do preenchimento, é necessário clicar no botão azul "continuar" para prosseguir a classificação de risco previamente selecionada.

\section{+ Paciente ACCR}

\begin{tabular}{|c|c|}
\hline Prontuário & \\
\hline 1610 & \\
\hline Convênio & \\
\hline Sistema Único de Saúde - SUS & \\
\hline Admissão & \\
\hline 04/03/2019 16:00 & \\
\hline Emergência & Não urgente \\
\hline
\end{tabular}

Fonte: Autor.

Figura G: Tela Riscos Assistenciais

A tela de riscos assistenciais está presente em dois ícones na tela menu paciente: módulos (ver figura N), são eles pronto atendimento e internação. É possível escolher a área em que o paciente está sendo admitido ou reclassificado. Esta tela oferece a opção de quatorze riscos assistenciais e três alertas de risco. O Enfermeiro deverá clicar a opção desejada e prosseguir a classificação. 
$\mathrm{Na}$ lateral direta da tela é possível verificar os riscos e alertas já classificados, em "itens selecionados", sendo possível mantê-los ou deletar em "limpar tudo!" antes da impressão da pulseira de identificação por cores. Esta funcionalidade tem o objetivo de garantir uma revisão ou atualização da classificação antes da impressão da pulseira.

Ao término do preenchimento, é necessário clicar no botão azul "gerar pulseira" para visualizar o layout da pulseira a ser impressa (ver figura Al) ou clicar no botão branco "cancelar, voltar" para abortar a classificação realizada.

\section{国 Riscos assistenciais}

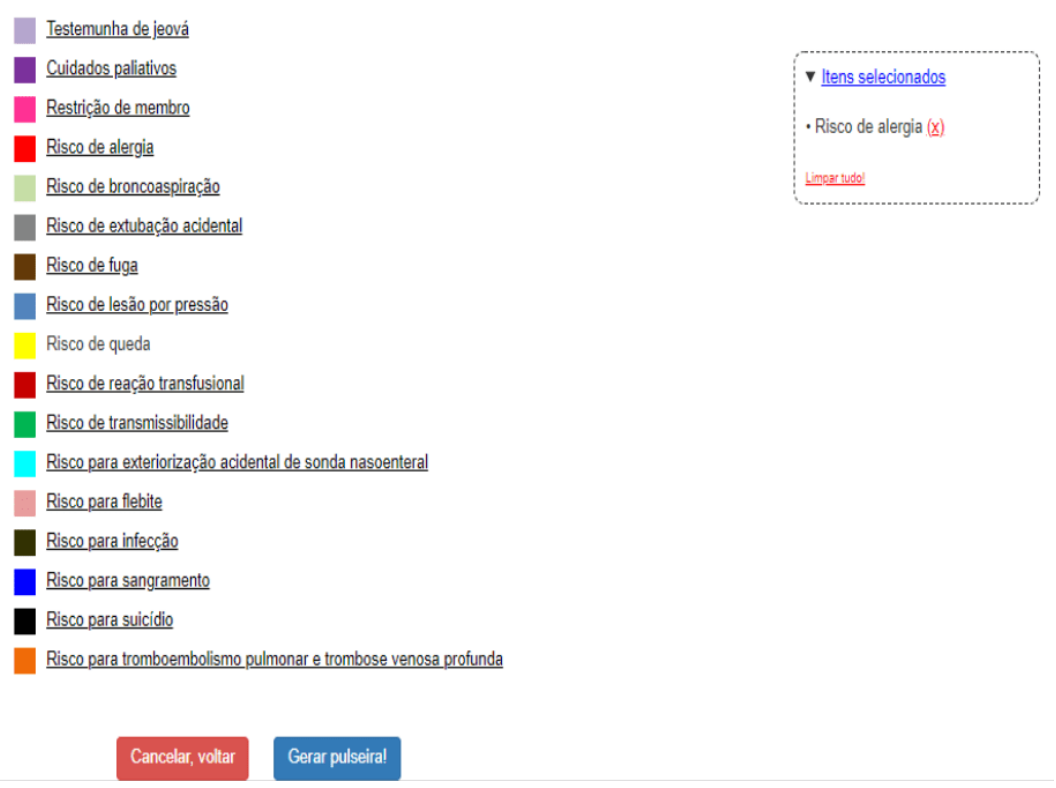

Fonte: Autor.

Figura H: Tela "visualizar pulseira"

A visualização da pulseira de identificação com tarjas coloridas de acordo com os riscos e alertas classificados se apresenta na tela de "riscos assistenciais" (ver figura M), o botão azul "gerar pulseira" foi programado para finalizar a classificação e resultar no produto para impressão.

$\mathrm{Na}$ lateral direita da tela é possível checar os riscos ou alertas escolhidos durante a avaliação do paciente e verificar a pulseira antes da impressão. Caso seja necessário 
algum acréscimo ou subtração de risco e/ou alerta é possível fechar a pulseira gerada

no botão vermelho

\section{$\mathrm{X}$} um risco e/ou alerta por vez da pulseira é necessário ir em "itens selecionados" e clicar

no símbolo

\section{(x)}

acréscimo de um risco e/ou alerta é só prosseguir a classificação do item desejado após o fechamento da pulseira.

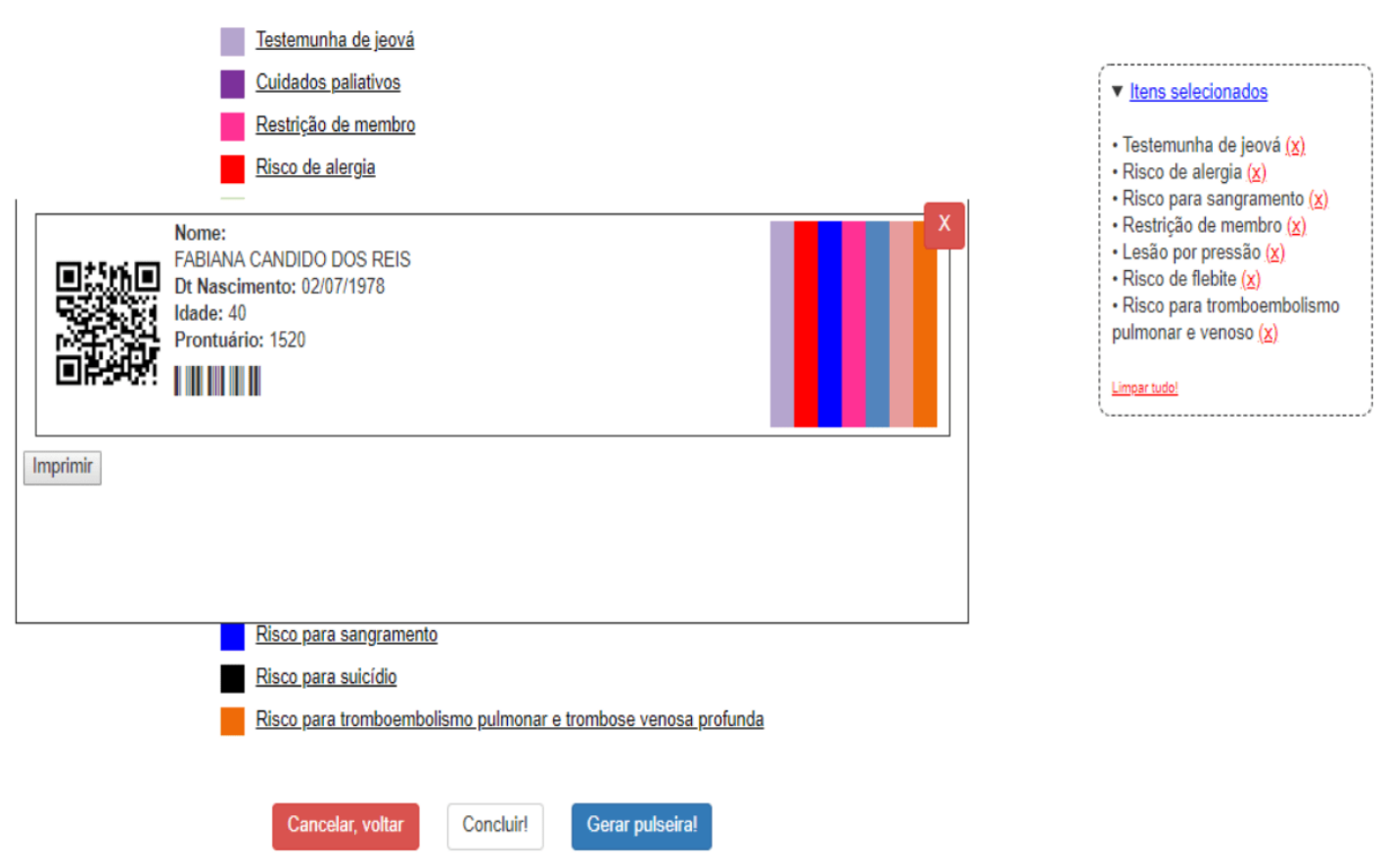

Fonte: Autor.

\section{CONSIDERAÇÕES FINAIS}

A segurança do paciente vem sendo motivo de discussões e estudos no Brasil e no mundo, e tornou-se o foco de todas as instituições de saúde.

A identificação do paciente é a primeira meta internacional de segurança do paciente e considerada primordial, quando seguido o protocolo corretamente, para evitar erros e incidentes que causem dano ao paciente. 
As pulseiras de identificação se tornaram usuais na maioria das instituições, principalmente hospitalares, pela facilidade de implantação e eficácia na identificação. Também são escolhas para alertar riscos e auxiliar na identificação de informações clínicas.

Porém ainda são muitas vezes utilizadas indevidamente, bem como as cores que sinalizam um risco ou informação clínica, não são padronizadas, facilitando a ocorrência do erro.

A tecnologia passou a ser utilizada em larga escala na segurança do paciente e gerenciamento de riscos. A tecnologia da informação e comunicação em saúde tornou-se fundamental na era da informática. Prontuários eletrônicos, colocados em sistemas e softwares complexos, padronizam e efetivam os registros, assegurando o acesso à informação e minimizando erros na comunicação multidisciplinar em larga escala.

Desta forma, criar um software que atenda à necessidade de padronizar e minimizar o erro na identificação do paciente, onde o enfermeiro classifica riscos e prescreve cuidados para estes riscos em bancos de dados eletrônicos e de fácil acesso à todos, bem como compilar todas as informações e alertas em uma única pulseira seria um grande passo na prestação de cuidados segura.

\section{REFERÊNCIAS}

AGÊNCIA NACIONAL DE VIGILÂNCIA SANITÁRIA (ANVISA). Rede sentinela histórico. Disponível em: http://www.anvisa.gov.br/servicosaude/hsentinela/historico.htm. Acesso em: 28 out. 2018.

AGÊNCIA NACIONAL DE VIGILÂNCIA SANITÁRIA. Assistência segura: uma reflexão aplicada à prática. Segurança do Paciente e Qualidade em Serviços de Saúde. Brasília: ANVISA, 2013. 
ASSOCIAÇÃO BRASILEIRA DE NORMAS TÉCNICAS (ABNT). ABNT NBR ISSO 31000. Gestão de riscos - Princípios e diretrizes. Rio de Janeiro: ABNT, 2010.

ASSOCIAÇÃO BRASILEIRA DE NORMAS TÉCNICAS. Tecnologia de informação Processos de ciclo de vida de software. Rio de Janeiro. 1998. Disponível em:

<http://aulasprof.6te.net/Arquivos_Aulas/06Qualidade_Soft/ABNT_NBR_ISO_12 207. $p d f>$ Acesso em: 18 jan.2019.

ÁVILA, A, M. Identificação por radiofrequência: tecnologia inteligente, hospital eficiente, qualidade e segurança para o paciente, 2012. Monografia (Especialização em Informação Científica e Tecnológica em Saúde) - Informação Científica e Tecnológica em Saúde, Fundação Oswaldo Cruz, Porto Alegre, 2012.

BAKKEN, S. S. et al. PHP Manual. Disponível em: <http://br.php.net/get/php_ manual_pt_BR.chm/from/this/mirror>. Acesso em: 20 mar. 2019.

BATTISTI, Júlio. Apostila de Hardware. Banco de Tutoriais do site Júlio Battisti, 2009. Disponível em: Acessado em 26 de abril de 2019.

BRASIL. Ministério da Saúde. Agência Nacional de Vigilância Sanitária. Resolução no 36, de 25 de julho de 2013. Implantação do Núcleo de Segurança do Paciente em Serviços de Saúde. Diário Oficial da União25 jul 2013; Seção 2. Disponível em: <http://bvsms.saude.gov.br/bvs//anvisa/2013.htm> Acesso em: 15 ago. 2018.

BRASIL. Ministério da Saúde. Gabinete do Ministro. Portaria MS/GM n 529, de 1 de abril de 2013. Institui o Programa Nacional de Segurança do Paciente (PNSP). Diário Oficial da União. 2013b. Disponível em: <http://bvsms.saude.gov.br/bvs/saudelegis/gm/2013/prt0529_01_04_2013.html> Acesso em: 15 ago. 2018.

BRASIL. Ministério da Saúde; Fundação Oswaldo Cruz; Agência Nacional de Vigilância Sanitária. Documento de referência para o Programa Nacional de Segurança do Paciente. Brasília - DF, 2014. Disponível em: 
<http://bvsms.saude.gov.br/bvs/publicacoes/documento_referencia_programa_nacio nal_seguranca.pdf> Acesso em: 06 ago. 2018.

CANDIDO, A. H. L; JUNIOR, KW. Design de produto e a pratica de construção de modelos e protótipos. Publicado em LDSM - Laboratório de Design e Seleção de Materiais.2009.

CONSELHO FEDERAL DE MEDICINA; SOCIEDADE BRASILEIRA DE INFORMÁTICA EM SAÚDE. Cartilha sobre Prontuário Eletrônico - A Certificação de Sistemas de Registro Eletrônico de Saúde. São Paulo, 2012. Disponível em: <http://portal.cfm.org.br/Cartilha_SBIS_Pront_Eletr.pdf> Acesso em: 10 set. 2018.

COSTA, V.T; MEIRELLES, B.H.S; ERDMANN, A.L. Melhores práticas do enfermeiro gestor no gerenciamento de risco. Rev. Latino-Am. Enfermagem, Ribeirão Preto, v. 21, n. 5, set/out. 2013.

DEGRACE, Peter; HULET, Leslie. Wicked problems, righteous solutions. USA: [s. n.], 1990.

EDWARDS, C. Anatomia e fisiologia dos computadores. In: HANNAH, Kathryn J.; BALL, Marion J.; EDWARDS, Margaret J.A. Introdução à informática em enfermagem. 3. ed, Porto Alegre: Artmed, p. 26-41, 2009.

FRAGATA, José; SOUSA, Paulo; SANTOS, Rui Seabra. Organizações de saúde seguras e fiáveis/confiáveis. In: SOUSA, Paulo; MENDES, Walter (org.). Segurança do Paciente: criando organizações de saúde seguras. 1. ed. Rio de Janeiro: FIOCRUZ, 2014. v. 2, cap. 1, p. 17-36. Disponível em: <http://books.scielo.org/id/vtq2b/cpub/sousa-9788575415948.epub> Acesso em: 10 set. 2018.

FASSINI, P; HAHN, G.V. Riscos à Segurança do Paciente em Unidade de Internação Hospitalar: Concepções da Equipe de Enfermagem. Rev. Enferm UFSM, Rio Grande do Sul, v. 2, n. 2, p. 290-299, Mai/Ago 2012. 
GIL, Antônio Carlos. Como elaborar projetos de pesquisa. 5 ed. São Paulo: Atlas, 2010.

GOMES, Adelia Quadros Farias. Iniciativas para segurança do paciente difundidas pela Internet por organizações internacionais: estudo exploratório, 2008. 135 f. Dissertação (Mestrado em Enfermagem) - Escola Nacional de Saúde Pública Sérgio Arouca ENSP. Rio de Janeiro, dezembro de 2008.

HOFFMEISTER, Louíse Viecili; MOURA, Gisela Maria Schebella Souto de. Uso de pulseiras de identificação em pacientes internados em um hospital universitário: aplicação na atenção hospitalar. Revista Latino-Americana de Enfermagem, Ribeirão Preto, v. 23, n. 1, jan/fev. 2015. Disponível em: <http://www.scielo.br/scielo.php?pid= \&script=sci_arttext\&tlng=pt> Acesso em: 10 set. 2018.

HOLLNAGEL, Elci. Proactive approaches to safety management. London: The Health Foundation; 2012.

International Civil Aviation Organization. Safety management. Annex 19 to the Convention on International Civil Aviation. Quebec: ICAO, 2013 Jul.

LAURENTI, Thaís Cristina et al. Gestão Informatizada de indicadores de úlcera por pressão. J. health inform, v.7, n.3, p. 94-8, set. 2015. Disponível em: <http://www.jhisbis.saude.ws/ojs-jhi/index.php/jhi-sbis/article/view/345> Acesso em: 31 ago. 2018.

LIMA, Helidéia de Oliveira; DUTRA, Elisabete Costa Reis. O gerenciamento de riscos na saúde: aplicação na atenção hospitalar. Revista de Administração Hospitalar e Inovação em Saúde, Minas Gerais, v. 4, n. 2, p. 87-90, 12 jul. 2010. Disponível em: <file:///C:/Users/Samsung/Downloads/1114-Texto\%20do\%20artigo-(1).pdf> Acesso em: 10 set. 2018.

KNAPP, Jack; ZERATSKY, John. SPRINT o método usado no Google para testar e aplicar novas ideias em apenas cinco dias. [S. I.]: Intrínseca, 2017. E-book. 
KRAUSE, Thomas R., HIDLEY, J.H. Taking the lead in patient safety: how healthcare leaders influence behavior and create culture. New Jersey: John Wiley \& Sons; 2009.

MAGALHÃES, Gildo. Introdução à metodologia da pesquisa: caminhos da ciência e tecnologia. São Paulo: Ática, 2005.

MAY, Chris Vander. Lições práticas sobre construções e lançamento de softwares aprendidas no dia a dia de trabalho no Google e Amazon. [S. I.]: Novatec, 2013. E-book.

MARQUES, Paulo Leonardo Ponte et al. Da pré-história à idade "mídia": a evolução da tecnologia no desenvolvimento da humanidade. In: SANTOS, Zélia Maria de Sousa Araújo; FROTA, Mirna Albuquerque; MARTINS, Aline Barbosa Teixeira. Tecnologias em Saúde: da abordagem teórica a construção e aplicação no cenário do cuidado. 1. ed. Fortaleza: EdUECE, 2016. cap. 1, p. 24-42.

MORIMOTO, Carlos E. Hardware. O Guia Definitivo. [S. I.: s. n.], 2014. E-book.

MORIMOTO, Carlos. Manual de Hardware completo. [S. I.: s. n.], 2015. E-book.

NASCIMENTO, João Costa; DRAGANOV, Patrícia Bover. História da qualidade em segurança do paciente. História da enfermagem: revista eletrônica, Portugal, v. 6, n. 2, p. 299-309, 28 nov. 2015. Disponível em: <http://here.abennacional.org.br/here/seguranca_do_paciente.pdf> Acesso em: 10 ago. 2018.

NEVES, Lourdes Alexandrina Castro; MELGAÇO, Regina Maria Tavares. A identificação do paciente como indicador de qualidade, 2011. Dissertação (Mestrado em Gestão de Ambiente e Segurança em Estabelecimentos Assistenciais de Saúde) - Universidade Federal do Estado do Rio de Janeiro. 2011. Disponível em: $<$ http://proqualis.net/dissertacao/o-paciente-como-indicador-de-qualidade> Acesso em: 30 ago. 2018. 
PARANAGUÁ, T.T.de. B; BRAGA, Q.de. P; BEZERRA, A.L.Q et al. Eventos adversos: instrumento de gerencia de la asistencia para la seguridad del paciente en el servicio de urgências, Enferm. glob, v. 13, n. 34, p. 206-23, abr. 2014.

PEREIRA, Déborah Santana et al. A tecnologia como ferramenta promotora da saúde. In: SANTOS, Zélia Maria de Sousa Araújo; FROTA, Mirna Albuquerque; MARTINS, Aline Barbosa Teixeira. Tecnologias em Saúde: da abordagem teórica a construção e aplicação no cenário do cuidado. 1. ed. Fortaleza: EdUECE, 2016. cap. 3, p. 64-82.

PEREIRA, Maria das Dores; SOUZA, Diego Floriano de; FERRAZ, Fabiane. Segurança do paciente nas ações de enfermagem hospitalar: uma revisão integrativa da literatura. Revista Inova Saúde, Criciúma, v. 3, n. 2, p. 123-156, 3 nov. 2014. Disponível em: <http://periodicos.unesc.net/article> Acesso em: 10 ago. 2018.

PINOCHET, Luis Hernan Contreras. Tendências de tecnologia de informação na gestão da saúde. O Mundo da Saúde, São Paulo, v. 35, n. 4, p. 382-394, 29 jan. 2011. Disponível em: <http://bvsms. saude.gov.br/bvs//tendencia.pdf> Acesso em: 10 set. 2018.

PRESSMAN, Roger S.; MAXIM, Bruce R. Engenharia de software: uma abordagem profissional. 7.ed. Porto Alegre: AMGH, 2011. Disponível em: $<$ https://pt.scribd.com/document/341109152/Engenharia-de-Software-UmaAbordagem-Profissional-pdf> Acesso em: 10 set. 2018.

QUIERELLE, D. A. Criando Sites com HTML-CSS-PHP. Construindo um projetoIniciante. São Paulo, p. 6-10, 2012. Disponível em: https://www.clubedeautores.com.br/book/126851-

Criando_sites_com_HTMLCSSPHP\#.WTyJmJLyvcs Acesso em: 22 jan. 2019.

REDE BRASILEIRA DE ENFERMAGEM E SEGURANÇA DO PACIENTE. Estratégias para a segurança do paciente: manual para profissionais da saúde. São Paulo, 2013. Disponível em: $<$ http://www.rebraensp.com.br/pdf/manualseguranca_paciente.pdf> Acesso em: 22 ago. 2018. 
RUBIN, Kenneth. SCRUM Essencial um guia prático para o mais popular processo ágil. [S. I.]: Alta Books, 2018. E-book.

SABBAGH, Rafael. SCRUM Gestão ágil para projetos de sucesso. São Paulo: [s. n.],

2017.Disponívelem:file://C:/Users/55119/Downloads/Scrum\%20Gestão\%20ágil\%20 para\%20projetos\%20de\%20sucesso\%20-\%20Casa\%20do\%20Codigo.pdf. Acesso em: 20 nov. 2018.

SANTOS, Sérgio Ribeiro dos. Informática em enfermagem: desenvolvimento de software livre com aplicação assistencial e gerencial. Rev Esc Enferm USP, São Paulo, v. 44, n. 2, p. 295-301, 2010. Disponível em: < http://www.ee.usp.br/reeusp> Acesso em: 30 ago. 2018.

SANTOS, Dartagnan Sousa dos. Segurança do paciente: implantação de pulseiras de identificação na uti adulto, 2014. 31 f. Monografia (Especialização em Enfermagem) - Universidade Federal de Santa Catarina. Florianópolis, 2014.

SÃO PAULO. Lei n. 10.241 de 17 de março de 1999. Dispõe sobre os direitos dos usuários dos serviços e das ações de saúde no Estado e dá outras providências. São Paulo: Assembléia Legislativa do Estado de São Paulo; 1999. Disponível em: <http://www.al.sp.gov.br/repositório/legislação/lei/1999> Acesso em: 10 set. 2018.

SKLAR, David. Learning PHP 5. [S. I.]: Reilly, 2004.

SOARES, Bruno. Aprendendo a linguagem PHP. São Paulo: Ciência Moderna, 2013. E-book.

SOMMERVILLE, I. Engenharia de Software [recurso eletrônico]. 9. ed. São Paulo: Pearson Prentice Hall, 2011.2 Disponível em: http://www.ifcamboriu.edu.br/ catia/IA16/Engenharia_Software_3Edicao.pdf Acesso em: 24 jan. 2019 
SOUSA, Paulino. Sistemas de Informação em Enfermagem: novos desafios, novas oportunidades. Rev. esc. enferm. USP, São Paulo. v. 46, n. 5, p. 1-2, out. 2012. Disponível em: <http://www.scielo.br/scielo.6001\&lng=pt\&nrm=iso> Acesso em: 31 ago. 2018.

SOUZA, Dalva Inês et al. Manual de orientações para projetos de pesquisa. Novo Hamburgo: Fundação Escola Técnica Liberato Salzano Vieira da Cunha, 2013.

SPERANDIO, Dircelene Jussara. Sistematização da Assistência de Enfermagem: Proposta de um software - protótipo, 2002. 75 f. Dissertação (Mestrado em Enfermagem) - Escola de Enfermagem de Ribeirão Preto da Universidade de São Paulo. Ribeirão Preto, 2002.

TORRES, Gabriel. Hardware e Redes. [S. I.: s. n.], 2010. E-book.

WATSON, D.S. Standardizing Wristband Colors. AORN J. v. 90, n. 3, p. 449-52, 2009.

WIKIPEDIA, Enciclopédia Livre. Microsoft Windows. Disponível em: Acessado em 05 de maio de 2019.

WORLD HEALTH ORGANIZATION. Collaborating Centre Patient Safety (Solutions). Washington, $2006 . \quad$ Disponível em: <http://www.who.int/patientsafety/Release.pdf> Acesso em: 07 ago. 2018.

WORLD HEALTH ORGANIZATION. Patient Safety Solutions Preamble, Genebra, Mai. 2007. Disponível em: <http://www.who.int/patientsafety/ patient safety /Preamble.pdf> Acesso em : 06 ago. 2018.

WORLD HEALTH ORGANIZATION. World Alliance for Patient Safety: forward programme. Genebra, $2005 . \quad$ Disponível em: <http://www.who.int/patientsafety/en/brochure_final.pdf> Acesso em: 06 ago. 2018.

WORLD HEALTH ORGANIZATION. World Alliance for Patient Safety, Taxonomy: The Conceptual Framework for the International Classification for Patient Safety: final technical report. Genebra, jan. 2009. Disponível em: 
$<$ http://www.who.int/patientsafety/taxonomy/icps_full_report.pdf> Acesso em: 07 ago. 2018.

Enviado: Julho, 2019.

Aprovado: Agosto, 2019. 